\title{
Performance of DNS as Location Manager for Wireless Systems in IP Networks
}

\author{
Abu Ahmed Sayeem Reaz, Mohammed Atiquzzaman, Shaojian Fu \\ Telecommunications and Networks Research Lab \\ School of Computer Science, University of Oklahoma, \\ Norman, OK 73019-6151, USA. \\ Email: $\{$ sayeem_reaz, atiq, sfu\}@ou.edu
}

Keywords: Location Management, DNS, Mobility Management, IP Diversity

Abstract-Domain Name System (DNS) can be deployed in the network as a Location Manager (LM) for mobility management. The suitability of Domain Name System (DNS) as an LM can be measured by how successfully it can serve to locate a mobile host. In this paper, we developed an analytical model to measure the performance of DNS as LM for mobility management techniques with IP Diversity support based on success rate which takes into account the radius of the subnet, the residence time of MH in that subnet, latency in the network and the overlapping distance of two neighboring subnets. Our analysis shows that for a reasonable overlapping distance, DNS can serve as an LM with very high success rate even under some high network latency.

\section{INTRODUCTION}

Increasing demand for mobility in wireless data networks has given rise to various mobility management schemes. Mobility management consists of two fundamental operations: Handoff and Location Management. Handoff occurs when a mobile device changes its point of attachment while still continuing with the service that it has been providing. In a layered network architecture for data communications, handoff can be managed at different layers. For example, Mobile IP (MIP) [1] is a network layer based handoff management scheme from IETF, MSOCKS [2] is a transport layer solution, and IEEE 802.11b follows a Layer 2 solution for handoff. Location management refers to finding valid the IP address a Mobile Host $(\mathrm{MH})$ by a Correspondent Node $(\mathrm{CN})$ in order to initiate and establish a connection.

There are two common choices for implementing a Location Manager (LM): Dedicated Location Manager, which is a separate network entity that serves as LM; and Domain Name System (DNS)[3], which provides name to IP mapping for locating a host in the Internet and supports dynamic secure updates [4]. DNS is a preferable option over dedicated LM because almost all connection establishments start with a name lookup from DNS [5] and it is already a part of the existing Internet infrastructure and unlike a dedicated LM, can be deployed without change in infrastructure. The
2922.

The research reported in this paper was funded by NASA Grant NAG3-
We investigate the suitability and performance of using DNS as an LM for mobility management as illustrated in Fig. 1 for a transport layer based mobility management scheme based on IP diversity. Having more than one interface cards is becoming

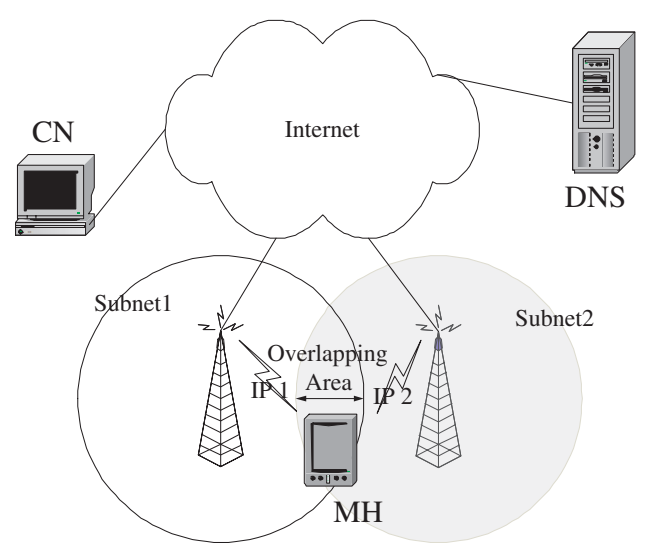

Fig. 1. DNS as a Location Manager.

common for mobile devices. During the handoff process, the $\mathrm{MH}$ has two IP addresses one for each of the neighboring subnets and communicates with both the APs at the same time with its multiple interface cards. This support for multiple IP address is called IP diversity, and our location management technique will be illustrated using a IP diversity enable mobility management scheme called Seamless IP diversity based Generalized Mobility Architecture (SIGMA) [6]. Each time a SIGMA enabled MH hands off to a new subnet, MH obtains a new IP address and it updates DNS with its new IP address (details in Sec. II-B).

Role of DNS as LM is to serve the correct IP address for $\mathrm{MH}$ and failure to do so results in a query failure. This type of failure can occur when a $\mathrm{CN}$ obtains an address from the LM, but the MH hands off to a new point of attachment before the connection request from the $\mathrm{CN}$ arrives at the $\mathrm{MH}$ due to delay in the network. Thus, the success rate of an LM is determined by the fraction of queries that result in a successful connection to the $\mathrm{MH}$.

One of the earliest suggestions on using directory server for location management can be found in [7]. Two proposals ([8], [9]) discuss the use of DNS as location manager. However, the 
authors are not aware of any work in the literature (including [7], [8], [9]) that consider performance evaluation or the challenges (such as query failure and higher traffic load) involved in using DNS as an LM in mobile data networks.

The objective of this paper is to analyze the performance of DNS as an LM based on success rate which takes into account the overlapping distance of two neighboring subnets, latency in the network, radius of the subnet and the residence time of $\mathrm{MH}$ in that subnet. Our contributions in this paper are (i) developing an analytical model to study the performance of DNS as LM, and (ii) identifying the impact of subnet residence time, overlapping distance, $\mathrm{MH}$ velocity and network delay on query failure. The result of our analysis shows that within reasonable $\mathrm{MH}$ velocity and network latency, DNS can be used as LM with a high success rate.

The rest of the paper is organized as follows. Sec. II describes the deployment of DNS as an LM, Sec. III develops the analytical model for evaluation of DNS as an LM. Sec. IV shows results on performance of DNS as LM, followed by conclusions in sec. V.

\section{DNS AND LOCATION MANAGEMENT}

Domain Name System represents a hierarchy of servers that includes Authoritative Name Server (ANS) that serves name to address mapping and Local Name Server (LNS) maintained at local networks that caches this mapping for a certain period of time (called Time To Live (TTL)) as indicated by the corresponding ANS for faster resolution of future queries.

\section{A. Deployment of DNS as LM}

The basic functionalities that LM requires to have [10] are (i) location update: whenever an $\mathrm{MH}$ changes its point of attachment, it will register the new IP address with the ANS via dynamic secure update [4]; (ii) location search: as DNS is invariant and almost ubiquitous connection originator, all connection from a $\mathrm{CN}$ will initially go to the ANS for name lookup; (iii) location search-update: each lookup query will be served with the new IP address reflecting the new location of the $\mathrm{MH}$ and $\mathrm{CN}$ would retrieve updated address from ANS.

In a mobile network, where $\mathrm{MH}$ is changing its IP address and updating DNS continuously, all the name lookup queries should be served by the ANS. Any non-zero TTL value suggested by an $\mathrm{MH}$ would make the ANS to instruct a querying LNS to cache the mapping for that TTL value and any new local request within that TTL period would be served by that LNS. But by that time MH might change its address again resulting in query failure. Therefore, TTL values at the ANS should be zero for all the MHs.

Challenges in deploying DNS as LM include higher traffic load on DNS which is insignificant with today's hardware advancement and safe delivery of update packets which is ensured through secure update proposed in [4].

The most significant challenge is during the handoff period. As shown in Fig. 2, when the DNS server is updated (due to handoff) at $t_{2}^{\prime}$ just after the $\mathrm{CN}$ has completed a query at $t_{1}^{\prime}$, the address obtained by $\mathrm{CN}$ may no longer be valid. The $\mathrm{CN}$ may not be able to find the MH when it sends a connection request at $t_{3}^{\prime}$. The effect of the above issue is minimized when

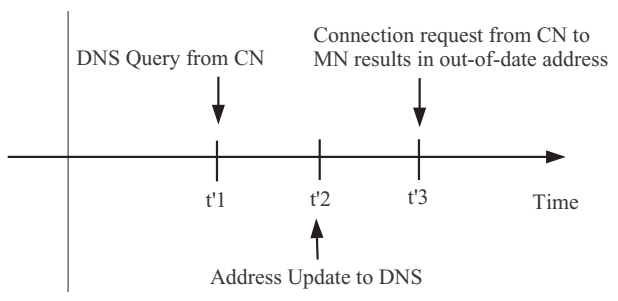

Fig. 2. Effect of obtaining out-of-date address by the CN. the handoff process is based on IP Diversity, as in SIGMA, which enables an $\mathrm{MH}$ to have two IP addresses and maintain two data streams during the handoff period. In that case, if the connection request arrives within the overlapping zone, even after the handoff, the $\mathrm{CN}$ would be able to locate the $\mathrm{MH}$ with old IP address.

\section{B. DNS as Location Manager for IP Diversity based Mobility Management}

We will illustrate the use of DNS as LM for an IP diversity based (e.g. SIGMA [6]) handoff scheme. During the residence of the $\mathrm{MH}$ in the overlapping area of the two neighboring subnets, the DNS record corresponding to an $\mathrm{MH}$ contains two IP addresses of the MH, and the DNS serves both the IP addresses in response to a location query. The order in which the two addresses are served determines the priority of the address during connection setup.

Fig. 3 shows the sequence of updates to the ANS by the $\mathrm{MH}$. At time $t_{1}, \mathrm{MH}$ enters overlapping area of neighboring subnets and obtains a new IP address. MH adds that address to ANS after the old one and subsequently ANS serves both the addresses. Then, at time $t_{2}, \mathrm{MH}$ hands off to the new subnet based on a threshold of relative signal strengths of the access points and changes the priorities of the addresses at ANS by putting the new one at higher order. At time $t_{3}$, MH leaves the overlapping area and removes the old IP from ANS.

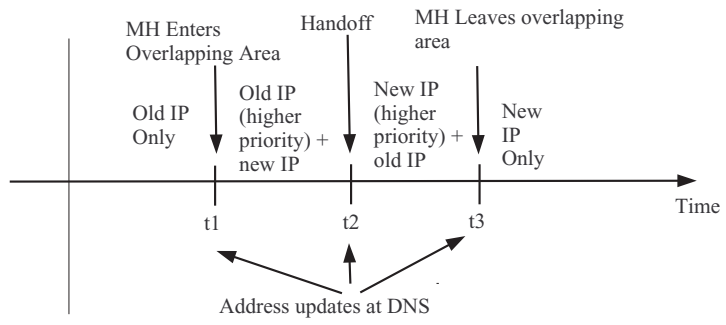

Fig. 3. MH's IP addresses in different stages of Handoff and their respective DNS updates.

\section{Analytical Model for PERformance EVALUATION OF DNS AS LM}

The primary success measure of a DNS as LM is how successfully it can provide the $\mathrm{CN}$ with the appropriate address such that the connection establishment request can be sent to the current address of the MH. We define success rate (Sec. 
III-C) as the fraction of queries successfully served out of the total number of queries. That can be represented by fraction of time where DNS might serve incorrect address, termed as the critical time (Sec. III-B), out of the total residence time (Sec. III-A).

\section{A. Calculation of Residence Time}

Mobile host moves according to Random Waypoint model [11], which is the most frequently used model in mobile networking research. In this mobility model, an $\mathrm{MH}$ randomly selects a destination point in the topology area according to uniform distribution, then moves towards this point at a random speed again uniformly selected between $\left(v_{\min }, v_{\max }\right)$. This one movement is called an epoch, and the elapsed time and the moved distance during an epoch are called epoch time and epoch length, respectively. At destination point, the $\mathrm{MH}$ will stay stationary for a period of time, called pause time, after that a new epoch starts.

Let,

$E(T)=$ expected value of epoch time.

$E(P)=$ expected value of MH pause time between movements.

$E(L)=$ expected value of epoch length.

$E(C)=$ expected number of subnet crossings per epoch.

$v=$ moving speed of $\mathrm{MH}$.

The objective of this section is to find the average residence time $\left(T_{\text {sub }}^{r e s}\right)$ for $\mathrm{MH}$ in a subnet. which can be estimated by the time between two successive movements (epoch time plus pause time) divided by the number of subnet crossings during this epoch, as shown in Eqn. (1):

$$
T_{s u b}^{r e s}=\frac{E(T)+E(P)}{E(C)}
$$

We first compute $E(T)$, since epoch length $L$ and movement speed $v$ are independent:

$$
E(T)=E(L / v)=E(L) E(1 / v)
$$

Since the moving speed is of uniform distribution between $\left(v_{\min }, v_{\max }\right)$, we have:

$$
\begin{aligned}
E(1 / v) & =\int_{v_{\min }}^{v_{\max }}(1 / v) \frac{1}{v_{\max }-v_{\min }} d v \\
& =\frac{\ln \left(v_{\max } / v_{\min }\right)}{v_{\max }-v_{\min }}
\end{aligned}
$$

Where $v_{\min }$ and $v_{\max }$ is minimum and maximum values of $v$.

In order to determine $E(L)$ and $E(C)$, we assume an arrangement of circular subnets in a rectangular topology as shown in Fig. 4, where $m, n$ are the number of vertically and horizontally arranged subnets in the topology, respectively. From [11], we know that $E(L)$ for a rectangular area of size $a \times b$ can be estimated as:

$$
\begin{gathered}
E(L)=\frac{1}{15}\left[\frac{a^{3}}{b^{2}}+\frac{b^{3}}{a^{2}}+\sqrt{a^{2}+b^{2}}\left(3-\frac{a^{2}}{b^{2}}-\frac{b^{2}}{a^{2}}\right)\right] \\
+\quad \frac{1}{6}\left[\frac{b^{2}}{a} \Phi\left(\frac{\sqrt{a^{2}+b^{2}}}{b}\right)+\frac{a^{2}}{b} \Phi\left(\frac{\sqrt{a^{2}+b^{2}}}{a}\right)\right]
\end{gathered}
$$

where $\Phi(\cdot)=\ln \left(\cdot+\sqrt{(\cdot)^{2}-1}\right)$.

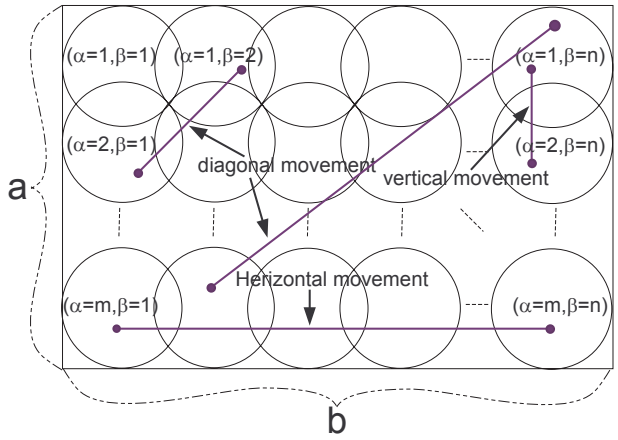

Fig. 4. Arrangement of subnets in a rectangular topology.

Now we can get epoch time $E(T)$ from Eqn.(2) using $M H$ velocity and epoch length obtained from Eqns. (3) and (4). Since pause time has been assumed to be uniformly distributed between $\left(0, P_{\max }\right)$, we have:

$$
E(P)=\int_{0}^{P_{\max }} \frac{P}{P_{\max }} d P=P_{\max } / 2
$$

Here $P_{\max }$ is the maximum pause time.

Next, we need to find $E(C)$, the general form of which can be expressed as [11]: $E(C)=$ $\frac{1}{m^{2} n^{2}} \sum_{\alpha_{j}=1}^{m} \sum_{\beta_{j}=1}^{n} \sum_{\alpha_{i}=1}^{m} \sum_{\beta_{i}=1}^{n} C\left(\begin{array}{c}\left(\alpha_{i}, \beta_{i}\right) \\ \left(\alpha_{j}, \beta_{j}\right)\end{array}\right)$

$C\left(\begin{array}{c}\left(\alpha_{i}, \beta_{i}\right) \\ \left(\alpha_{j}, \beta_{j}\right)\end{array}\right)$ is the number of subnet crossings caused by one movement between subnet $\left(\alpha_{i}, \beta_{i}\right)$ to $\left(\alpha_{j}, \beta_{j}\right)$, which depends on the actual subnet shape and arrangement. Consider the circular subnet arrangement as shown in Fig. 4, we can observe three kind of movements: horizontal, vertical and diagonal. $C\left(\begin{array}{c}\left(\alpha_{i}, \beta_{i}\right) \\ \left(\alpha_{j}, \beta_{j}\right)\end{array}\right)$ can be generalized by the following Manhattan distance metric:

$C\left(\begin{array}{c}\left(\alpha_{i}, \beta_{i}\right) \\ \left(\alpha_{j}, \beta_{j}\right)\end{array}\right)=\left|\alpha_{i}-\alpha_{j}\right|+\left|\beta_{i}-\beta_{j}\right|$

So, we can get the expression for $E(C)$ :

$$
E(C)=\frac{1}{m^{2} n^{2}} \sum_{\alpha_{j}=1}^{m} \sum_{\beta_{j}=1}^{n} \sum_{\alpha_{i}=1}^{m} \sum_{\beta_{i}=1}^{n}\left(\left|\alpha_{i}-\alpha_{j}\right|+\left|\beta_{i}-\beta_{j}\right|\right)
$$

Substituting epoch time, pause time and subnet crossing from Eqns. (2), (5) and (6) into Eqn. (1), we can get the expression for $T_{s u b}^{r e s}$.

\section{B. Calculation of Critical Time}

For analytical tractability, we make the simplifying assumption that all the queries are processed at the ANS without any referrals. Then the process of communication initiation between an $\mathrm{MH}$ and $\mathrm{CN}$ has two parts. First the $\mathrm{CN}$ gets the Name to IP address mapping from the ANS, and then it initiates a connection with the MH with the IP as illustrated by the timeline in Fig. 5 .

We denote $\Delta t_{1+2}=t_{2}-t_{1}$ and $\Delta t_{2+1}=t_{3}-t_{1}$ as illustrated in Fig. 3. Here $\Delta t_{1+2}$ is the time during which MH is in the overlapping area when the first address has a 


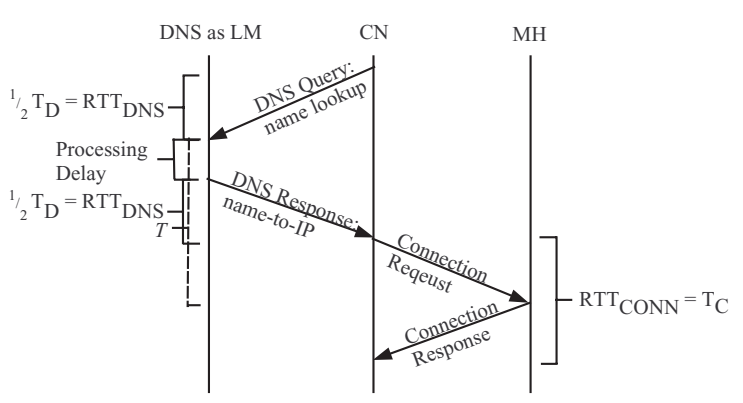

Fig. 5. Timeline of connection initiation from $\mathrm{CN}$ to $\mathrm{MH}$.

higher priority, i.e., before the handoff, and $\Delta t_{2+1}$ is the time spent by $\mathrm{MH}$ in overlapping area when the new address has a higher priority, i.e., after the handoff. Let

$$
\tau=\left(\frac{1}{2} T_{D}\right)+\left(\frac{1}{2} T_{C}\right)+T_{S}^{d}
$$

Here $\frac{1}{2} T_{D}$ represents the time taken by the DNS name lookup reply to come from $\mathrm{ANS}$ to $\mathrm{CN}, \frac{1}{2} T_{C}$ represents the time taken by the connection establishment request from $\mathrm{CN}$ to $\mathrm{MH}$ and, $T_{S}^{d}$ is the query processing delay at ANS.

If the residency time of an $\mathrm{MH}$ in the overlapping area is $\Delta t_{1+2}+\Delta t_{2+1}$, for a DNS query to be successfully served with the current IP address of $\mathrm{MH}$

$$
\tau \leq\left(\Delta t_{1+2}+\Delta t_{2+1}\right)
$$

Now, in the internet, the round trip delay is sum of round trip propagation delay, transmission delay and queuing delay. If

$T_{C A}^{d}=$ Propagation delay between $\mathrm{CN}$ and ANS

$T_{C M}^{d}=$ Propagation delay between $\mathrm{CN}$ and $\mathrm{MH}$

$\beta_{C A}=\mathrm{BW}$ of the link between $\mathrm{CN}$ and ANS

$\beta_{C M}=\mathrm{BW}$ of the link between $\mathrm{CN}$ and $\mathrm{MH}$

$\psi_{D}=$ Avg. DNS query packet size

$\psi_{C}=$ Avg. connection request packet size

$\bar{\xi}=$ Avg. queuing delay in the network

$\frac{1}{2} T_{D}=T_{C A}^{d}+\frac{\psi_{D}}{\beta_{C A}}+\bar{\xi}$

and $\frac{1}{2} T_{C}=T_{C M}^{d}+\frac{\psi_{C}}{\beta_{C M}}+\bar{\xi}$

Therefore,

$$
\tau=T_{C A}^{d}+T_{C M}^{d}+\frac{\psi_{D}}{\beta_{C A}}+\frac{\psi_{C}}{\beta_{C M}}+2 \bar{\xi}+T_{S}^{d}
$$

If the latency in the network increases, value of $\tau$ would increase and violate Eqn. (8). Then if $\tau>\left(\Delta t_{1+2}+\Delta t_{2+1}\right)$,

$$
T_{c r}=\left(\tau-\left(\Delta t_{1+2}+\Delta t_{2+1}\right)\right)
$$

where any location query made within time $T_{c r}$ would carry a possibility of failure. We call this period Critical Time.

Now, if $d_{s u b}$ is radius of a subnet and $d_{o v r}$ is the overlapping distance, the asymptotic density function that gives the probability of the $\mathrm{MH}$ to be at a certain point on a line segment $[0$, $\left.d_{\text {sub }}\right]$ is given by $f_{x}(x)=-\frac{6}{d_{s u b}^{3}} x^{2}+\frac{6}{d_{s u b}^{2}} x$ where $\mathrm{x}$ is any point on the line segment which basically reflects the distance of the MH from the center of the subnet [11]. Thus, Probability of an $\mathrm{MH}$ being within that subnet is $\int_{0}^{d_{\text {sub }}} f_{x}(x) d x=1$ and Probability of the $\mathrm{MH}$ being in the overlapping zone is $\int_{x_{\text {min }}}^{d_{\text {sub }}} f_{x}(x) d x=1+2\left(\frac{x_{\text {min }}}{d_{\text {sub }}}\right)^{3}-3\left(\frac{x_{\text {min }}}{d_{\text {sub }}}\right)^{2}$ where $x_{\text {min }}=$ $d_{\text {sub }}-d_{\text {ovr }}$. Then if $T_{\text {ovr }}^{\text {res }}$ is the residence time of $\mathrm{MH}$ in the overlapping zone, then $T_{\text {ovr }}^{r e s}=T_{\text {sub }}^{r e s} \int_{x_{\text {min }}}^{d_{\text {sub }}} f_{x}(x) d x$.

From Eqn. (7), essentially,

$$
T_{\text {ovr }}^{r e s}=\left(\Delta t_{1+2}+\Delta t_{2+1}\right)=T_{\text {sub }}^{r e s} \int_{x_{\text {min }}}^{d_{\text {sub }}} f_{x}(x) d x
$$

Network latency, $\tau$ and residence time in overlapping area,

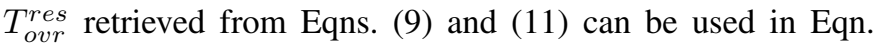
(10) to get critical time, $T_{c r}$.

\section{Calculation of Success Rate}

Now, we can find the number of failures during a single handoff as $\mathrm{E}\left[\chi\left(T_{c r}\right)\right]$ and total number of queries as $E\left[\chi\left(T_{\text {sub }}^{r e s}\right)\right]$ where $\chi(t)$ represents number of queries within time $t$. If $\lambda$ is the arrival rate of name lookup query to the LM, we have $E\left[\chi\left(T_{c r}\right)\right]=\lambda T_{c r}$ and $E\left[\chi\left(T_{s u b}^{r e s}\right)\right]=\lambda T_{s u b}^{r e s}$.

The success of DNS as an LM, depends on the fraction of time it can successfully serve the right IP address out of all the queries. So, Success Rate, $\rho$, can be defined as

$$
\rho=\frac{E\left[\chi\left(T_{s u b}^{r e s}\right)\right]-E\left[\chi\left(T_{c r}\right)\right]}{E\left[\chi\left(T_{\text {sub }}^{r e s}\right)\right]}
$$

Subnet residence time and critical time from Eqns. (1) and (11) are used to get success rate, $\rho$ from Eqn. (12).

\section{RESULTS}

Eqn. (12) determines that the success rate for DNS as LM which depends on residence time of $\mathrm{MH}$ in a subnet and the critical time. Critical time is dependant on the latency in the network and the residence time of $\mathrm{MH}$ in the overlapping region. Residence time depends on subnet radius and mobile velocity. So, in short, the success rate depends on latency in the network, velocity and overlapping distance between subnets.

One of the performance measures is how the success rate varies over different overlapping distances for different network latencies. For an average epoch length $L=300$ meters, average $\mathrm{MH}$ velocity $v=20 \mathrm{~m} / \mathrm{sec}$, maximum pause time $P_{\max }=10 \mathrm{sec}$, and for $10 \%$ of MHs changing subnet during an epoch (Eqn. (1)), a very high processing delay at server $T_{S}^{d}$ $=3 \mathrm{sec}$ and subnet radius $d_{s u b}=500$ meter, if the overlapping distance varies between 0 and 40 meters and if network latency varies from 0.3 to 1.8 seconds (Eqn. (10)), we found out that for an overlapping distance of about 30 meters (or above), the success rate remains one as illustrated in Fig. 6.

For the same configuration, if we have a fixed network latency of $0.5 \mathrm{sec}$ while a varying $T_{S}^{d}$ from 0.1 to $3.1 \mathrm{sec}$ (Eqn. 9), we see from Fig. 7 that for an overlapping distance over 40 meters, which is only $5 \%$ of the subnet diameter, the success rate settles to one even with a high $T_{S}^{d}$ of $3.1 \mathrm{sec}$.

Another performance measurement variable is the residence time of $\mathrm{MH}$ in the subnet. How quickly an $\mathrm{MH}$ crosses a subnet and an overlapping region determines the residence 


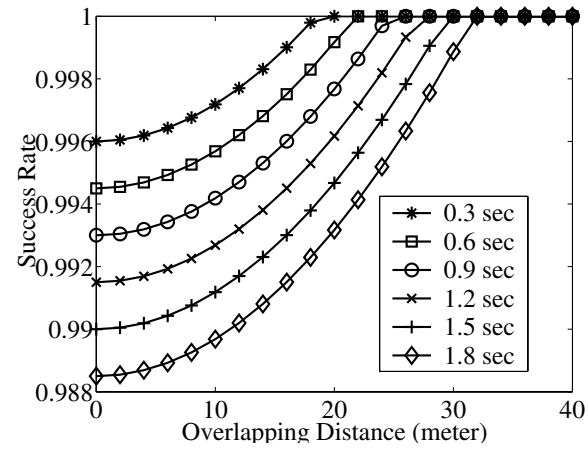

Fig. 6. Success rate against overlapping area for different network latency.

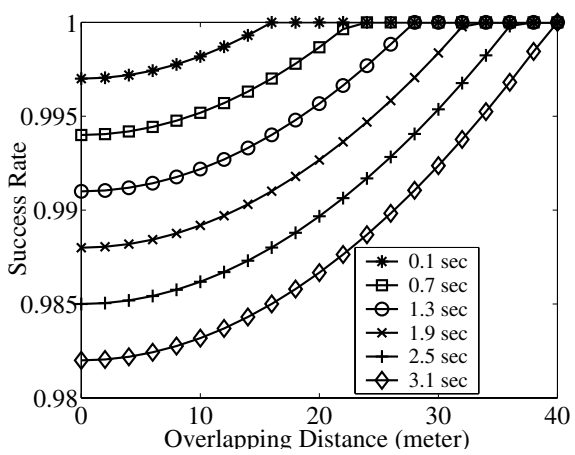

Fig. 7. Success rate against overlapping area for different query processing time at LM.

time of $\mathrm{MH}$ in the subnet $\left(T_{s u b}^{r e s}\right)$ and in the overlapping region $\left(T_{o v r}^{r e s}\right)$, respectively. This is basically a function of $\mathrm{MH}$ velocity $v$. So, for a given latency of the 0.5 seconds in the network and $T_{S}^{d}=3 \mathrm{sec}$, if the overlapping distance varies within 0 and 40 meters and if $v$ (Eqn 3) varies from $10 \mathrm{~m} / \mathrm{sec}$ to $60 \mathrm{~m} / \mathrm{sec}(60 \mathrm{~m} / \mathrm{sec}=135 \mathrm{miles} / \mathrm{hour})$, we found out that for $d_{\text {ovr }}=30$ meters (or above), the success rate remains one. Fig. 8 shows how success rate changes over overlapping distance with varying residence time.

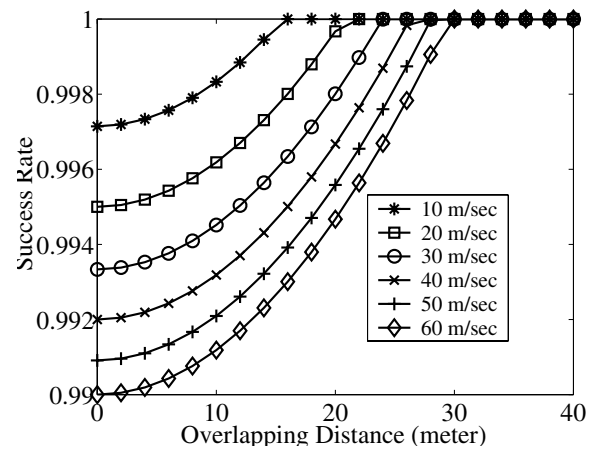

Fig. 8. Success rate against overlapping area for different $\mathrm{MH}$ velocity.

Now, as residence time of MH in both subnet and overlapping area is dependant on the subnet radius, if all the determinant factors of $T_{s u b}^{r e s}$ and $\tau$ remains static, then $T_{c r}$ would depend on $T_{o v r}^{r e s}$. If $d_{s u b}$ varies, then the relative overlapping would vary and so would $T_{\text {ovr }}^{\text {res }}$. So, for a given $\tau$ of the 2 seconds in the network, if the overlapping distance varies within 0 and 40 meters, $T_{s u b}^{r e s}$ remains at $600 \mathrm{sec}$ and $d_{\text {sub }}$ varies from 250 meters to 750 meters, we found out that for $d_{\text {ovr }}=30$ meters (or above), we find that success rate remains one. Fig. 9 depicts the effect of subnet radius on varying overlapping area.

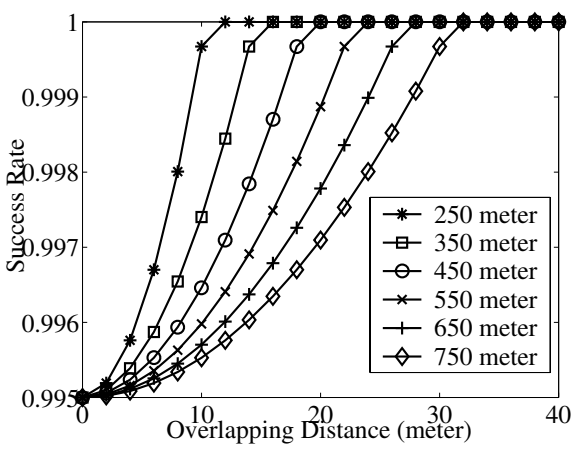

Fig. 9. Success rate against overlapping area for different subnet radius.

Thus we can conclude that within reasonable latency and overlapping region, DNS would be able to serve as an LM successfully.

\section{Conclusions}

DNS has been proposed for location management in literature. Although DNS is widely deployed in the Internet, the authors are not aware of any prior work on the performance of DNS as LM in mobile data networks. In this paper, we developed an analytical model to study the performance of DNS as LM in terms of success rate, Internet traffic load and subnet radius. Our results clearly shows that DNS can serve as a location manager without query failure even with only $5 \%$ overlapping distance between two subnets. We can conclude that DNS is a feasible solution for location management in mobile data networks.

\section{REFERENCES}

[1] C. Perkins, "IP mobility support." IETF RFC 3344, August 2002.

[2] D. A. Maltz and P. Bhagwat, "MSOCKS: an architecture for transport layer mobility," IEEE INFOCOM, San Francisco, CA, pp. 1037 - 1045, March 29 - April 2, 1998.

[3] P. Mockapetris, "Domain name - implementation and specification." IETF RFC 1035, November 1987.

[4] D. E. Eastlake, "Secure domain name system dynamic update." IETF RFC 2267, January 1998.

[5] J. Jung, E. Sit, H. Balakrishnan, and R. Morris, "DNS performance and the effectiveness of caching," IEEE/ACM Transactions on Networking, vol. 10, no. 5, pp. 589-603, October 2002.

[6] S. Fu, L. Ma, M. Atiquzzaman, and Y. Lee, "Architecture and performance of SIGMA: A seamless handover scheme for data networks," IEEE ICC, Seoul, South Korea, May 16-20, 2005.

[7] B. Awerbuch and D. Peleg, "Concurrent online tracking of mobile users," Computer Communication Review, vol. 21, no. 4, pp. 221-233, September 1991.

[8] D. Funato, K. Yasuda, and H. Tokuda, "TCP-R: TCP mobility support for continuous operation," IEEE International Conference on Network Protocols, Atlanta, GA, pp. 229 - 236, October 28 - 31, 1997.

[9] A. C. Snoeren and H. Balakrishnan, "An end-to-end approach to host mobility," MOBICOM, Boston, MA, pp. 155 - 166, August 6-11, 2000.

[10] P. Krishna, N. H. Vaidya, and D. K. Pradhan, "Location management in distributed mobile environments," Conference on Parallel and Distributed Information Systems, Austin, TX, pp. 81 - 88, September 1994.

[11] C. Bettstetter, H. Hartenstein, and X. Prez-Costa, "Stochastic properties of the random waypoint mobility model," Wireless Networks, vol. 10, no. 5, pp. 555-567, September 2004. 